\title{
Magnetic field as a characterization of wide and narrow spaces in a real challenging scenario using Dynamic Time Warping
}

\author{
Antoni Perez-Navarro*†, Raul Montoliu ${ }^{\ddagger}$, Joaquín Torres-Sospedra ${ }^{\ddagger}$, and Jordi Conesa*§ \\ ${ }^{*}$ Faculty of Computer Science, Multimedia and Telecommunication, \\ Universitat Oberta de Catalunya (UOC), Rambla del Poblenou, 156, 08018 Barcelona, Spain \\ ${ }^{\dagger}$ Internet Interdisciplinary Institute (IN3 - UOC), Av. Carl Friedrich Gauss, 5, 08860 Castelldefels, Barcelona, Spain \\ $\ddagger$ Institute of New Imaging Technologies, Universitat Jaume I, 12071 Castellón, Spain \\ $\S$ eHealth Center (eHc - UOC), Avinguda del Tibidabo, 39-43, 08035, Barcelona, Spain
}

\begin{abstract}
This paper presents a study of indoor positioning in public zones of the Parc Taulí Hospital in Sabadell. It is a challenging scenario because: 1) it combines wide spaces with middle sized and narrow spaces; 2 ) it is a shielded zone where no signals are available, and therefore, no WiFi signal can be used for positioning; and 3) it is not possible to deploy beacons for positioning. The goal of this work is to test whether it is possible to get indoor positioning in a real and challenging scenario by using only the magnetic field. The positioning precision requires to locate the part of the hospital where the user is. The proposed solution defines "virtual corridors" to improve positioning in wide areas. To validate the work, magnetic field data have been recorded from the scenario, using different smartphones and by different persons. The obtained magnetic data curves have been compared by using dynamic time warping distance. Results show that it is possible to characterize every path with the magnetic field. The main contributions of the present paper are: 1) defining "virtual corridors" as a way to position using magnetic field in 2D spaces; and 2) showing that even in wide spaces, like the hall of a hospital, it is possible to find magnetic anomalies linked to positions.
\end{abstract}

Index Terms-Android, Magnetic field, Indoor Positioning, Proof of Concept, Challenging Scenarios.

\section{INTRODUCTION}

Nowadays, mobile devices are widely used for navigation purposes. Commercialization of smartphones, that have positioning systems, Global Navigation Satellite Systems (GNSS) and Internet, have allowed to develop a large set of applications based on localization, most commonly known as Location Based Systems (LBS) and Context Aware Recommender Systems (CARS). [1] Nevertheless, those applications usually work only outdoors since most of them are based on GNSS, which signal is too low in indoor environments.

Indoor location, despite the efforts and advances of last years, [2] is still an open field that is far from a universal system like GNSS are. Nowadays several indoor applications for specific purposes can be found, but there is not any universal system that allows positioning within indoor environments, such as in a hospital, in the same way that GNSS allows to position in different cities anywhere in the world by using different universal applications, like Google Maps or Apple Maps.
There is not even consensus regarding the best mechanism to get indoor positioning. The two main types of systems are: infrastructure free, that includes systems able to position only with the technology carried by the user like inertial systems or positioning via magnetic field; and infrastructure based, that position using information from sensors and emitters associated to the space. In this last group, we can also distinguish two kinds of systems: dedicated, that deploy sensors or emitters which purpose is positioning, like those systems using beacons [3]-[6]; and not dedicated, that use opportunity signals which are there for other purposes, like WiFi [7], or cellular networks.

Regarding the algorithms for positioning, although they depend on the kind of system chosen, usually several different algorithms can be applied for every single system. Inertial systems use pedestrian dead reckoning (PDR) techniques; systems based in infrastructure can use trilateration or fingerprinting, among others; and among them, systems with dedicated infrastructure can implement their own mechanisms of localization, some of which emulate the GNSS mechanisms. This diversity enables the adoption of custom deployments in real world problem, where one or more algorithms are combined to provide robust positioning.

Why is still so open the world of indoor positioning? The answer to this question is wide but we can focus it in two main aspects: 1) every single building introduces specific particularities and challenges; and 2), every single application has its own requirements of positioning. Regarding the building, it is very different to get positioning in a wide open space like an airport or in the hall of a hospital, than to get positioning in a space formed by narrow corridors and offices. Regarding the application requirements, it is very different an application that needs millimetre precision, like a robot guidance system, than an application that only needs to say in which part of the building the user is; and it is also very different an application addressed to very specific users, than one addressed to the general public, that should have to deal with the user's devices, mainly smartphones. Therefore, the building and the application will condition the system and algorithms to choose. 
It is worth to note that the building can also condition the positioning system, not only because of its own features, but also because of restrictions due of usage or owners. The building is, in fact, the main difference with respect to GNSS. GNSS is a universal system whose signals can be received in outdoor spaces (except if they cannot because of physical reasons, like urban canyons). Contrary to GNSS, every single building is like a small isolated world, with its own features.

In the present paper we show the case of Parc Taulí Hospital $(\mathrm{PTH})^{1}$ in the city of Sabadell, $30 \mathrm{~km}$ north of Barcelona (Spain). The problem to solve with indoor positioning is to know the zone of the building where the user is. Therefore, finegrained precision is not an application requirement. However, PTH is a very challenging scenario because of several reasons:

- Its building contains both wide spaces, like the main hall and reception; and corridors combined with middle size spaces, like those corridors that go to the visit rooms, and through waiting rooms.

- It is a WiFi free zone: following the World Health Organization recommendations regarding electromagnetic fields [8], no WiFi signal is present in the hospital. But also, no emitters can be added, to avoid the presence of electromagnetic fields. Therefore, it is not possible to install beacons.

- Electromagnetic fields are shielded: usually hospitals have many devices which emit strong electromagnetic fields. Nevertheless, there are teams of physics and engineers for shielding the fields these devices generate.

Taking all these restrictions into account, we propose using an infrastructure free positioning indoor system. Within this group, the main systems are inertial and based on magnetic field. In this paper we test whether a system based on magnetic field is suitable for getting the zone with the user is in PTH.

Although the devices are electromagnetically shielded, the terrestrial magnetic field is still present. A recent work shows how to model the ambient magnetic field [9], and from the results it can be seen that the presence of the building is enough to introduce changes in the magnetic field. Nevertheless, will be the variations of this magnetic field enough to characterize the different spaces to deal with? I.e. will we be able to associate some features to a single space? This is the question that the present paper faces.

The paper is structured as follows: first of all, an state of the art is presented, focused mainly in solutions regarding magnetic field positioning. Then, the methodology followed to record and analyse data is presented and, finally, the results and conclusions are discussed.

\section{STATE OF THE ART}

Most of smartphones are equipped with GPS and GLONASS, but also with several other sensors, like WiFi, bluetooth, gyroscope, magnetometer. We can also find some smartphones equipped even with UWB sensors, thermometer and barometer. As it can be seen on [10], this constellation of sensors has driven

\footnotetext{
${ }^{1}$ http://www.tauli.cat/hospital/
}

to different techniques and systems for $2 \mathrm{D}$ indoor positioning in private networks. Among the most popular are those that use WiFi sensor, because WiFi is widespread and WiFi access points are very convenient as beacons for indoor positioning.

Nevertheless, last years, positioning via magnetic field is gaining rellevance [11]-[15]. It is convenient because geomagnetic field is present everywhere, and no infraestructure is needed, dedicated or not. Positioning may take into account the distortions of the geo-magnetic field produced by metallic parts of buildings [16] or any device, like lifts or vending machines. Thus, positioning can be achieved just with a smartphone.

Previous work has shown the temporal stability of magnetic field in indoor environments [17], and repetitiveness of measures, i.e. measures are similar when taken in the same point at different times. Other works have shown that repeating the experiment with different smartphones give similar results [14].

The main drawback of magnetic field positioning is the low variability of measures. Several techniques have been used to solve this problem: Monte Carlo techniques to choose the more logical next point in a path [17]; or Hidden Markov Model (HMM) [13], among others.

Another item to take into account is which part of the magnetic field consider, since it is a three dimensions vector. Some authors propose the module of the magnetic field [11]; others propose use only the $Z$ component, that is more stable [13]; and other authors use statistical information extracted from the module or every component [12], [14].

To get position, the most common approximation is fingerprinting. [18]-[21] This kind of algorithms are divided into two phases: off-line phase and on-line phase. In off-line phase the magnetic map of the indoor environment is created. This map associate a position with a value of the magnetic field. In the on-line phase, users obtain measures with their device and, comparing their values with the fingerprints, the algorithm is able to get the position.

In WiFi fingerprinting, values are usually obtained from a single position during the off-line phase. Since the data received is an scalar, the influence of the orientation of the smartphone is lower. Nevertheless, when dealing with magnetic field, in all previous works, values from single points have not been taken, but the shape of the curve in a path passing through the points. Since magnetic field is a vector, this means that every single fingerprint will depend on the time required to take it and the device orientation. Due to the importance of capturing data, several proposals have been proposed for the off-line phase: using an automatic system [14]; using potential users of the application, which allows to get data associated to common paths [19]; or using a rolling platform to get data from every direction [12]. However, despite the system used to get the reference data, in the on-line phase users will measure data.

It is important to note, that magnetic field is mainly used in one-dimensional scenarios, like corridors [14], [17]; and is only scarcely used in two-dimensional scenarios [13]. Previous works warn about the difficulties of using magnetic field in wide environments [21], and other studies alert about the difficulties of using magnetic field in old buildings, where little metallic 
structures exist [22]. As a conclusion, magnetic fields are mainly used to get position in a path and not in a single position, although some works use the reference points [12], [23].

\section{Methodology}

As has been stated previously, the goal of the paper is to establish whether the magnetic field could be an appropriate signal to find position in the PTH environment.

Figure 1 shows the floor plan of PTH. In the figure, several zones are marked: zone $A$ is the hall entrance, where reception and information desk is located, and gives access to zone $B$ and zone $C$; zone $B$ is a second hall that grant access to the lifts and to another corridor; zone $C$ is a corridor that gives access to some waiting rooms, to a bathroom, the vending machines and two lifts; and zone $D$ gives access to several waiting rooms. As can be seen from the plan, zone $A$ and zone $B$ are big wide areas, while zone $C$ and zone $D$ are narrow areas (corridors) combined with small wide areas (waiting rooms).

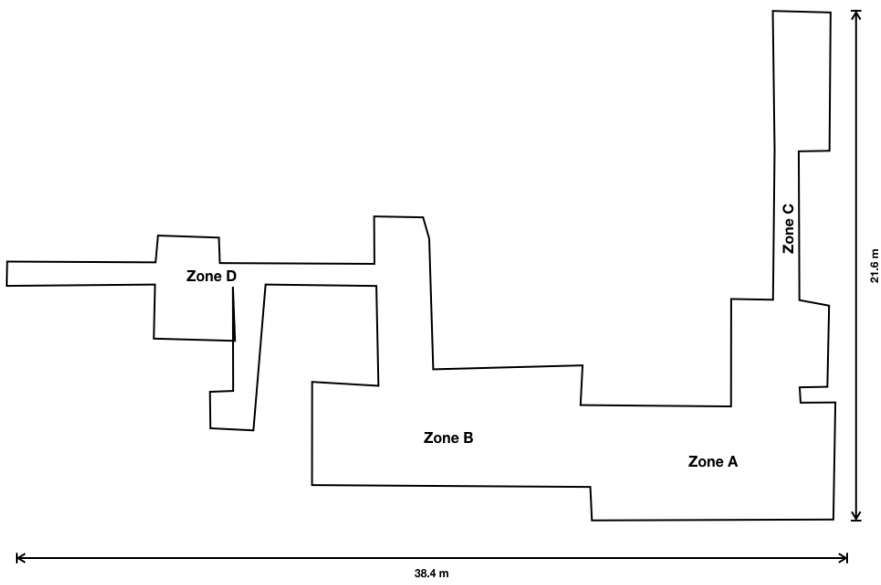

Fig. 1. Schematic floor plan of the zone to position in Parc Taulí Hospital in Sabadell.

Figures 2 and 3 show a detail of the reference elements that can be found in zones $\mathrm{A}$ and $\mathrm{C}$, respectively.

As has been seen in the state of the art section, magnetic field value is highly dependant on the sensor and therefore it is not possible to associate a value to a single point, but the shape of the curve to a line, i.e. the magnetic field can be taken as reference when we have a set of values in a path. The shape of the curve is the reference to be taken. This is so because the key to use the magnetic field as a reference are the anomalies introduced by metallic elements in the geomagnetic field and the magnetic field produced by fixed devices. Although this could seem a limitation, in fact makes the magnetic field very appropriate for walking users in corridor-like zones since, in this kind of spaces, users use to walk always by the same path. Magnetic field is, therefore, suitable for lineal spaces, that can be called 1D spaces, like zones $\mathrm{C}$ and $\mathrm{D}$, but not for 2D spaces like zones A and B. Although the motion patterns of people is a complex topic [24], [25], since the goal of this work is characterize zones, we will restrict the study to linear

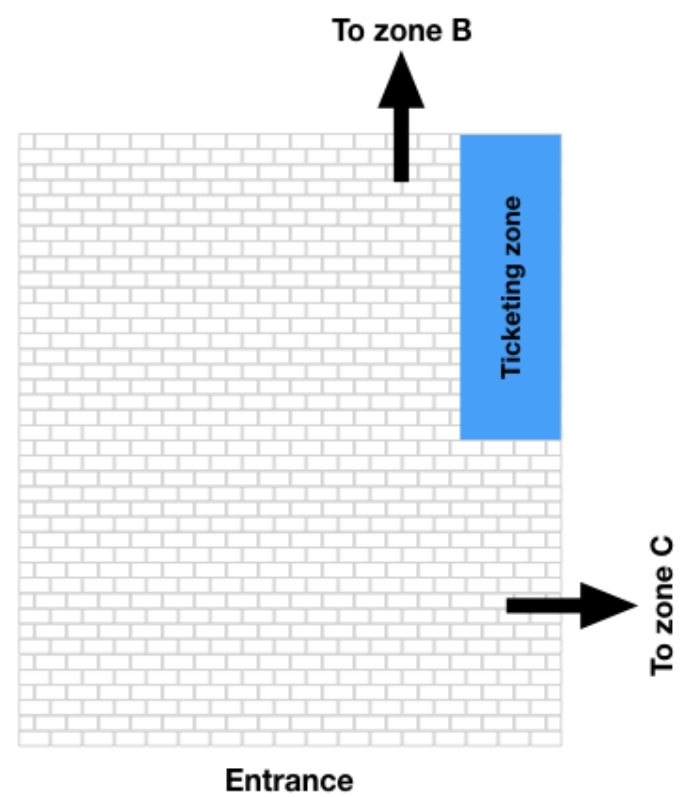

Fig. 2. Detail of Parc Taulí Hospital in Sabadell: Hall next to the entrance in zone A. It covers an area of $10.2 \times 12 \mathrm{~m}^{2}$

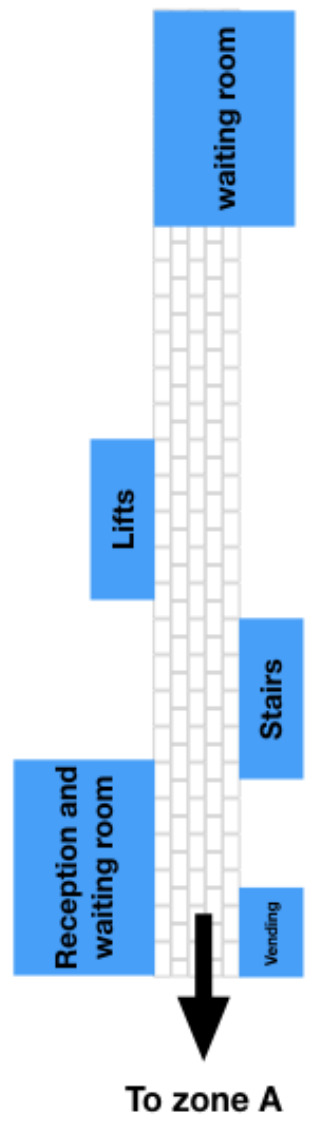

Fig. 3. Detail of Parc Taulí Hospital in Sabadell: Corridor in zone C. It covers an area of $8.1 \times 3.0 \mathrm{~m}^{2}$ 
movements. Therefore, how can be zones A and B transformed in lineal zones?

One approach is to discretise the underlying 2D indoor space as a graph as done by the Arara's team in [2]. In practice, it corresponds to assign the possible locations to nodes and the edges connect neighbour nodes.

To answer this question, we identified the usual routes that users from PTH follow when arriving to the hospital. Some reference points are identified: information/reception desk, ticketing machines and lifts. Thus allows to identify which routes that users can follow get into the hospital:

1) From the entrance, users can go to:
a) the reception/information desk
b) the ticketing machines
c) the lifts
d) zone $\mathrm{C}$
e) zone D

2) From the reception/information desk, users can go to:
a) the ticketing machines
b) the lifts
c) zone $\mathrm{C}$
d) zone D

3) From the ticketing machines, users can go to:
a) the reception/information desk
b) the lifts
c) zone $\mathrm{C}$
d) zone D

This information allows to identify "virtual corridors" [26] in zones A zone B and emulate 1D spaces suitable for magnetic field positioning in these spaces.

Nevertheless, wide 2D spaces have another drawback for positioning with magnetic field. What allows to associate a path to a magnetic field are the magnetic features that are present in that path. These features are because of devices in fixed positions and anomalies in the geomagnetic field introduced by building structures. Narrowness of corridors help to make these features available for magnetometers, however, in wide spaces like zone $A$ and zone $B$, walls are far from the paths and devices are next to the wall. Thus, it is important to remember that the main question with which the present paper deals is, if it will be possible to associate a magnetic curve to a single path. It is important to note that hospital authorities only need to know in which zone the user is.

\section{A. Data collection}

Thus, to get magnetic data in a way that let us answer the question if magnetic field allows us to identify where the user is, the procedure to get data is as follows:

- 5 smartphones with different versions of Android have been used: two Nexus 5, a Samsung Galaxy A3, a Samsung Galaxy A5 and a Samsung Galaxy S3. Having data from the same smartphone carried by different users, can give information about the effect of users and it is possible to check the effect of the device.
- The data were provided as logfiles recorded by a dedicated Android application, GetSensorData v2 developed by CSIC, which is available from [27] and has been prolifically used [2], [28], [29]. This application gets data from magnetometer, accelerometer, gyroscope and $\mathrm{WiFi}$ sensors and allows to indicate the location of landmarks.

- Users have followed the "virtual corridors" previously indicated in both directions. Figure 4 shows the route followed and the points marked as reference. The paths recorded are:

- Path 1: $1 \rightarrow 2$,

- Path 2: $2 \rightarrow 1$,

- Path 3: $1 \rightarrow 3 \rightarrow 4$,

- Path 4: $4 \rightarrow 3 \rightarrow 1$,

- Path 5: $1 \rightarrow 5$,

- Path 6: $5 \rightarrow 1$,

- Path 7: $1 \rightarrow 6$,

- Path 8: $4 \rightarrow 6$,

- Path 9: $1 \rightarrow 7$,

- Path 10: $4 \rightarrow 7$,

- Path 11: $6 \rightarrow 1$,

- Path 12: $7 \rightarrow 1$,

- Path 13: $1 \rightarrow 8$,

- Path 14: $1 \rightarrow 9$,

- Path 15: $4 \rightarrow 8$,

- Path 16: $4 \rightarrow 9$.

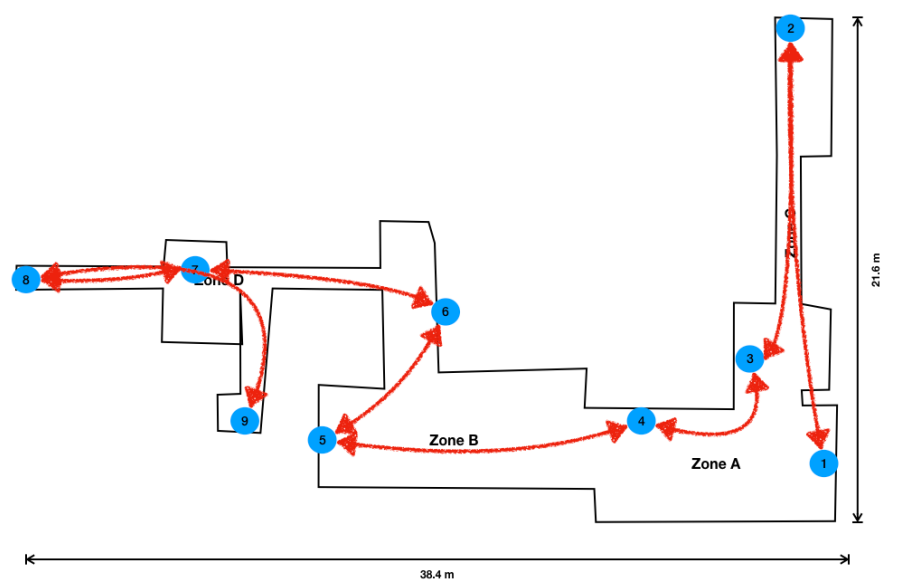

Fig. 4. Detail of the routes taken in the Parc Taulí Hospital. The reference points (or landmarks) are also shown in the figure. 16 different paths have been recorded.

Since data from every path is taken twice, in both directions, we have 16 different paths taken. Although the application takes data from the three axes of the magnetic field, we will only take the value of the module because it is independent of the rotation of the device. Figure 5 shows the changes of the magnetic field by axis, while the module remains stable.

\section{B. Data analysis}

To analyse data, records from every single smartphone are divided by paths. Since our goal is to show whether it is possible to characterize a path with the magnetic field, to maximize 


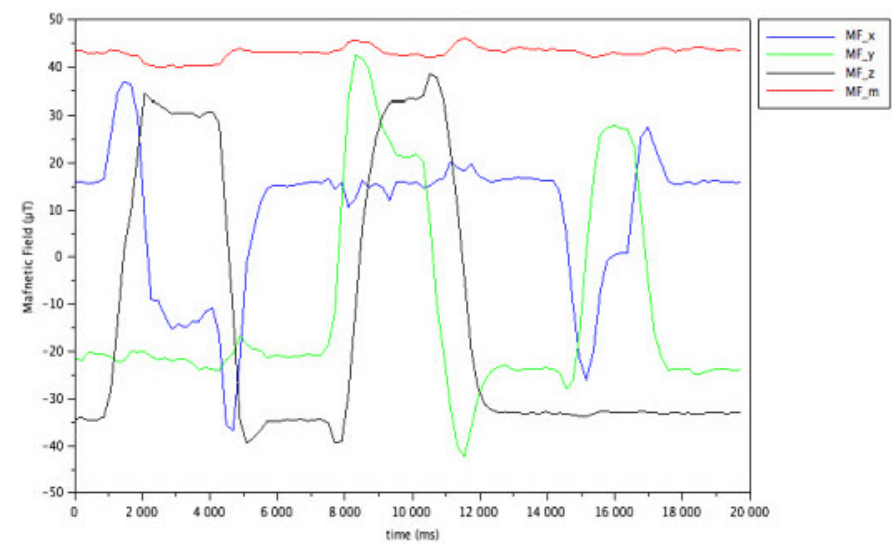

Fig. 5. Changes on magnetic field when rotating the smartphone $360^{\circ}$

testing, we will compare every path obtained from every single smartphone, with all the paths from the other smartphones. It is important to note that for every smartphone one set of data is used for learning, and data from the other smartphones is used for testing. This will allow to compare every path with 64 paths (16 paths*4 smartphones), which corresponds to a leaving-one-device-out cross-validation strategy. If the magnetic field measured by every single smartphone characterize the path, the closest path to everyone will be the corresponding path from the other files.

To compare paths, we obtain the distance between them with a Dinamic Time Warping (DTW) algorithm. This algorithm allows to compare the shape of curves, even if the time period is different for every one of them. Thus, if the magnetic field characterizes a path, the shape of the curve obtained in function of the time by different users, should have to be the same, regardless the time needed to walk along the path. Therefore, DTW will show that these two curves are close one to the other. The calculation of distances has been performed by using the DTW library of the software R [30], [31].

\section{RESUlts}

Before showing the results, the magnetic field curves are shown for visual inspection. Figure 6 show the module of the magnetic field versus time for all the paths of one single smartphone. As can be seen, those paths that correspond to the same part, but are taken in the opposite direction, are specular images from each other. Look for example the path 01 and 02 or path 15 and 16 . This is a first check pointing out that magnetic field might characterize a zone. However, paths 3 and 4 or paths 5 and 6 looks different, although the general tendency is similar. These paths are the ones that go to the zones where many people is waiting for something: point 3 is the information desk, point 4 is where the ticketing machines are and point 5 is where the lifts are. The presence of people can affect the path followed when taking data, in a different way when arriving to the point, than when leaving from it.

The next step is finding out the distance for every path to the paths obtained from the other smartphones. As it has been said before, this information has been obtained by calculating the distance using the DTW algorithm. In tables I to V they are shown, as an example, the distances between one path obtained with every smartphone, and the DTW distance to the paths obtained with the other smartphones. "DS $n$ " means distance to the corresponding path of smartphone $n$. Thus, every row of the table shows the distance from the path obtained with the smartphone corresponding to that table, to the path of the row, obtained with every other smartphone. The shortest distance for every smartphone is shown in bold letter. As can be seen, the shortest distance always corresponds to the operational path. i.e. for table I, that is calculated for path 2 with smartphone 1 , the shortest distance to the paths obtained with the other smartphones is with path 2 of every smartphone; and the same happens with the other tables.

TABLE I

DTW DISTANCES BETWEEN DATA FROM PATH 2 AND SMARTPHONE 1 TO ALL THE PATHS OBTAINED WITH THE OTHER SMARTPHONES. "DS $n$ " MEANS DISTANCE TO THE CORRESPONDING PATH OF SOURCE $n$.

\begin{tabular}{|c|c|c|c|c|}
\hline Path & DS1 & DS2 & DS3 & DS4 \\
\hline 1 & 59222.556 & 69096.932 & 66288.812 & 52288.714 \\
\hline $\mathbf{2}$ & $\mathbf{2 0 0 8 . 7 7 6}$ & $\mathbf{1 8 6 2 . 3 6 2}$ & $\mathbf{1 8 5 9 . 1 7 7}$ & $\mathbf{1 4 4 3 . 7 4 8}$ \\
\hline 3 & 46731.020 & 47054.183 & 38020.907 & 35159.075 \\
\hline 4 & 124838.325 & 122441.871 & 116202.213 & 90248.133 \\
\hline 5 & 180367.699 & 169678.142 & 172101.845 & 150649.214 \\
\hline 6 & 250454.185 & 252585.450 & 256104.203 & 216115.899 \\
\hline 7 & 289314.198 & 298745.292 & 296995.854 & 257449.608 \\
\hline 8 & 513961.733 & 552622.412 & 538330.248 & 425265.575 \\
\hline 9 & 708924.420 & 738334.175 & 733827.290 & 578625.718 \\
\hline 10 & 593981.091 & 633179.344 & 622692.547 & 533186.535 \\
\hline 11 & 747916.029 & 718870.812 & 736233.979 & 617133.404 \\
\hline 12 & 677813.581 & 712522.454 & 721966.666 & 622556.896 \\
\hline 13 & 963233.572 & 940669.116 & 979589.967 & 814875.963 \\
\hline 14 & 880372.307 & 935202.446 & 838019.921 & 746889.570 \\
\hline 15 & 1196016.691 & 1165210.181 & 1235164.594 & 1025146.911 \\
\hline 16 & 1408420.809 & 1449350.990 & 1462299.793 & 1203575.653 \\
\hline
\end{tabular}

TABLE II

DTW DISTANCES BETWEEN DATA FROM PATH 5 AND SMARTPHONE 2 TO ALL THE PATHS OBTAINED WITH THE OTHER SMARTPHONES. "DS $n$ " MEANS DISTANCE TO THE CORRESPONDING PATH OF SOURCE $n$.

\begin{tabular}{|c|c|c|c|c|}
\hline Path & DS1 & DS2 & DS3 & DS4 \\
\hline 1 & 291852.797 & 289376.738 & 295445.286 & 220389.863 \\
\hline 2 & 180367.699 & 190798.728 & 179550.837 & 142193.289 \\
\hline 3 & 79043.618 & 73612.695 & 76641.841 & 63542.104 \\
\hline 4 & 42163.613 & 36545.325 & 60569.072 & 32819.572 \\
\hline $\mathbf{5}$ & $\mathbf{1 2 6 9 . 0 4 1}$ & $\mathbf{1 4 6 7 . 2 8 5}$ & $\mathbf{2 0 9 3 . 2 9 8}$ & $\mathbf{1 4 8 4 . 9 3 2}$ \\
\hline 6 & 15995.448 & 18260.221 & 19243.281 & 14118.812 \\
\hline 7 & 59005.809 & 61628.586 & 61674.983 & 49140.216 \\
\hline 8 & 184425.677 & 202873.082 & 195023.607 & 140452.915 \\
\hline 9 & 331363.065 & 352356.641 & 348367.408 & 253672.826 \\
\hline 10 & 275687.582 & 295798.751 & 288496.918 & 228433.953 \\
\hline 11 & 372858.235 & 356855.950 & 369275.957 & 285472.211 \\
\hline 12 & 337156.052 & 350753.827 & 357742.198 & 285506.906 \\
\hline 13 & 516437.970 & 520536.515 & 550271.238 & 426294.961 \\
\hline 14 & 450064.361 & 512730.737 & 436330.053 & 365983.816 \\
\hline 15 & 752377.253 & 690905.668 & 747411.156 & 581460.750 \\
\hline 16 & 955494.132 & 916867.860 & 927368.522 & 716964.732 \\
\hline
\end{tabular}

These results can be found if all the possible tables are build. Therefore we found that the closer paths to $100 \%$ of the paths obtained with one smartphone, are the corresponding 


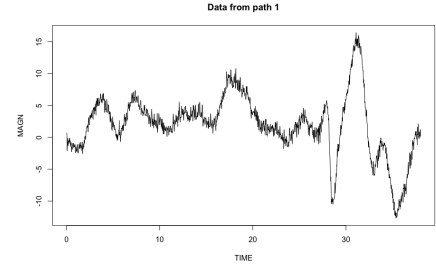

Path 01

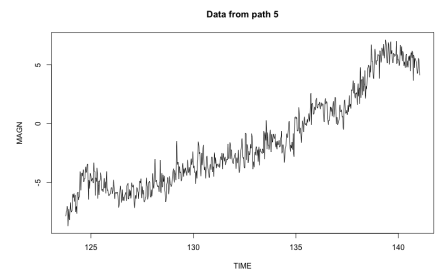

Path 05

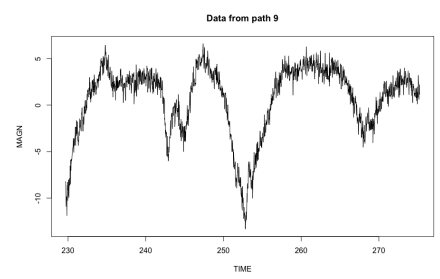

Path 09

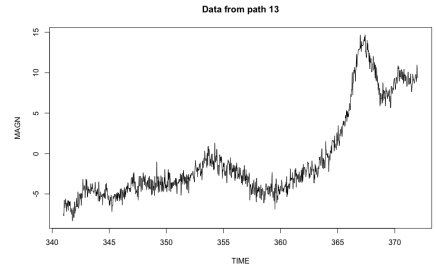

Path 13

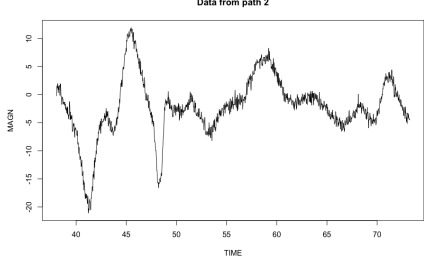

Path 02

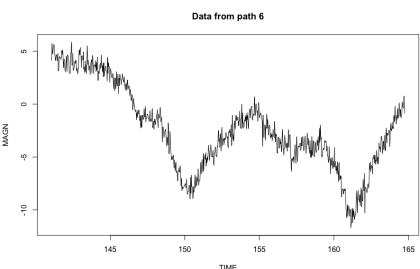

Path 06

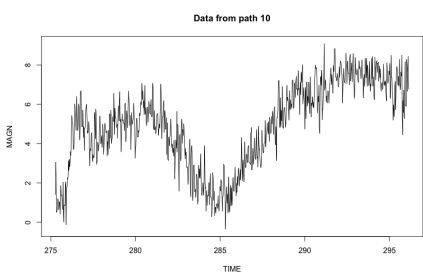

Path 10

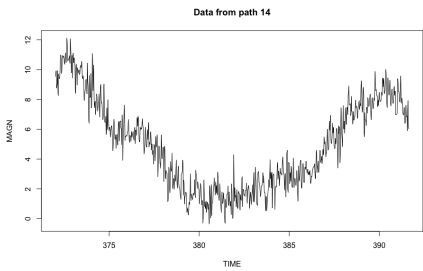

Path 14

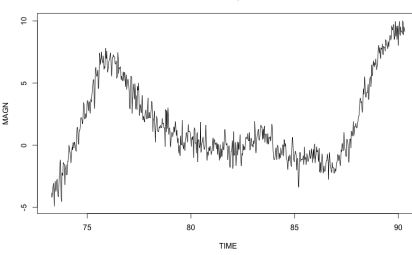

Path 03

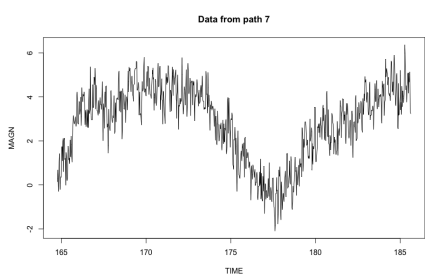

Path 07

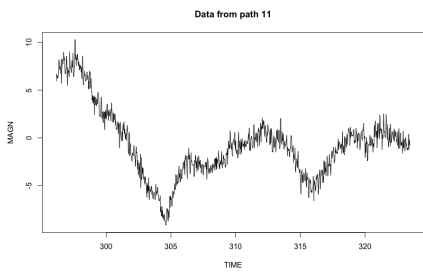

Path 11

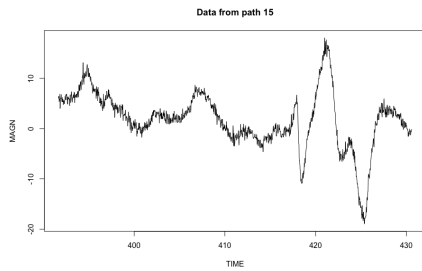

Path 15

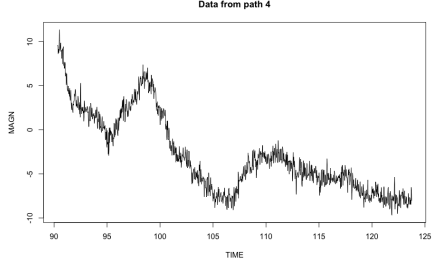

Path 04

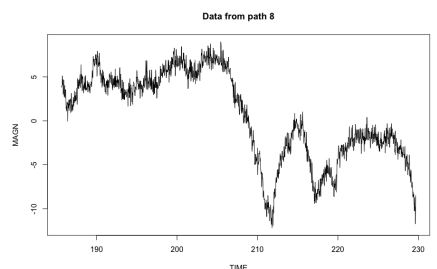

Path 08

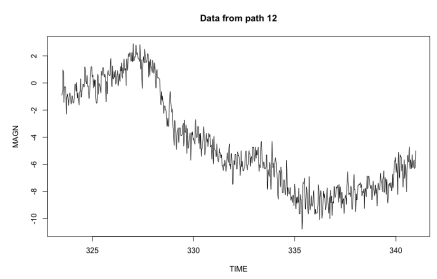

Path 12

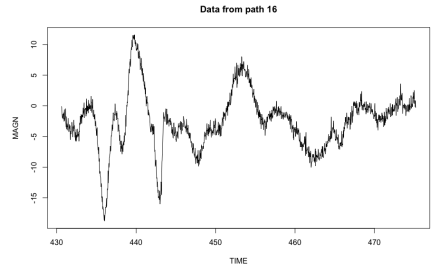

Path 16

Fig. 6. Module of the magnetic field versus the time for the 16 paths.

TABLE III

DTW DISTANCES BETWEEN DATA FROM PATH 9 AND SMARTPHONE 3 TO ALL THE PATHS OBTAINED WITH THE OTHER SMARTPHONES. "DS $n$ "

MEANS DISTANCE TO THE CORRESPONDING PATH OF SOURCE $n$.

\begin{tabular}{|c|c|c|c|c|}
\hline Path & DS1 & DS2 & DS3 & DS4 \\
\hline 1 & 913912.780 & 949397.189 & 921438.154 & 771013.171 \\
\hline 2 & 738334.175 & 711962.741 & 737840.472 & 640900.196 \\
\hline 3 & 515741.594 & 500956.439 & 505703.859 & 460563.785 \\
\hline 4 & 520152.814 & 506668.043 & 531819.204 & 441458.728 \\
\hline 5 & 356550.928 & 352356.641 & 365534.585 & 328853.336 \\
\hline 6 & 309812.239 & 296805.907 & 312372.643 & 273690.091 \\
\hline 7 & 226948.428 & 217163.059 & 218135.420 & 197381.938 \\
\hline 8 & 121666.007 & 122815.356 & 113082.533 & 94971.106 \\
\hline $\mathbf{9}$ & $\mathbf{2 4 2 5 . 3 2 4}$ & $\mathbf{2 7 3 8 . 0 3 5}$ & $\mathbf{1 0 7 1 . 6 3 1}$ & $\mathbf{1 1 3 1 . 6 8 4}$ \\
\hline 10 & 48751.988 & 46539.315 & 60350.815 & 55973.516 \\
\hline 11 & 130133.159 & 126911.230 & 142220.474 & 124935.434 \\
\hline 12 & 198665.366 & 193821.313 & 211968.064 & 190562.348 \\
\hline 13 & 311326.613 & 315408.976 & 336870.794 & 289917.632 \\
\hline 14 & 364880.227 & 375760.334 & 372870.160 & 341491.914 \\
\hline 15 & 564302.374 & 551422.883 & 571995.216 & 489502.412 \\
\hline 16 & 783668.242 & 739545.222 & 773515.862 & 654501.051 \\
\hline
\end{tabular}

TABLE IV

DTW DISTANCES BETWEEN DATA FROM PATH 12 AND SMARTPHONE 4 TO ALL THE PATHS OBTAINED WITH THE OTHER SMARTPHONES. "DS $n$ "

MEANS DISTANCE TO THE CORRESPONDING PATH OF SOURCE $n$.

\begin{tabular}{|c|c|c|c|c|}
\hline Path & DS1 & DS2 & DS3 & DS4 \\
\hline 1 & 862144.21 & 926423.59 & 851293.741 & 659997.216 \\
\hline 2 & 721966.67 & 694911.10 & 775948.403 & 574725.415 \\
\hline 3 & 439689.11 & 425527.10 & 433201.185 & 347186.029 \\
\hline 4 & 564665.26 & 551177.92 & 543712.307 & 422895.381 \\
\hline 5 & 351000.57 & 357742.20 & 341297.375 & 298421.456 \\
\hline 6 & 365960.85 & 353972.15 & 380071.379 & 300537.163 \\
\hline 7 & 298775.80 & 276949.95 & 291679.536 & 230335.357 \\
\hline 8 & 359400.20 & 354801.11 & 368501.738 & 267841.519 \\
\hline 9 & 223857.67 & 226902.25 & 211968.064 & 159254.194 \\
\hline 10 & 79319.35 & 80021.74 & 69628.129 & 58154.290 \\
\hline 11 & 33621.89 & 36091.10 & 22798.521 & 20407.367 \\
\hline $\mathbf{1 2}$ & $\mathbf{3 8 2 6 . 7 3}$ & $\mathbf{2 4 6 9 . 2 7}$ & $\mathbf{1 1 7 2 . 8 8 9}$ & $\mathbf{9 0 8 1 . 4 1 4}$ \\
\hline 13 & 24104.83 & 23987.66 & 31189.291 & 26387.422 \\
\hline 14 & 70449.55 & 75438.15 & 87885.912 & 64295.266 \\
\hline 15 & 181005.90 & 174662.63 & 176751.019 & 144494.382 \\
\hline 16 & 328970.17 & 299594.34 & 316459.909 & 247266.697 \\
\hline
\end{tabular}


TABLE V

DTW DISTANCES BETWEEN DATA FROM PATH 15 AND SMARTPHONE 5 TO ALL THE PATHS OBTAINED WITH THE OTHER SMARTPHONES. "DS $n$ " MEANS DISTANCE TO THE CORRESPONDING PATH OF SOURCE $n$.

\begin{tabular}{|c|c|c|c|c|}
\hline Path & DS1 & DS2 & DS3 & DS4 \\
\hline 1 & 1186013.17 & 1265462.465 & 1172591.581 & 1201255.5063 \\
\hline 2 & 1025146.91 & 992735.776 & 1093247.077 & 1026842.1603 \\
\hline 3 & 675052.76 & 657494.210 & 667310.191 & 649425.2210 \\
\hline 4 & 845826.35 & 829358.455 & 819249.263 & 853417.1808 \\
\hline 5 & 571265.73 & 581460.750 & 558264.930 & 594679.8712 \\
\hline 6 & 600800.69 & 585687.668 & 621217.289 & 627873.6918 \\
\hline 7 & 517367.09 & 486194.558 & 507917.354 & 499990.0393 \\
\hline 8 & 643721.53 & 635857.203 & 663095.125 & 650398.2052 \\
\hline 9 & 499200.09 & 503264.440 & 489502.412 & 497053.6668 \\
\hline 10 & 263209.36 & 261643.348 & 255971.608 & 257678.9980 \\
\hline 11 & 231071.00 & 236678.121 & 207937.344 & 217217.8033 \\
\hline 12 & 147846.41 & 147247.370 & 143060.211 & 144494.3816 \\
\hline 13 & 113499.15 & 117763.610 & 103885.860 & 101814.9827 \\
\hline 14 & 42072.30 & 38852.090 & 30187.421 & 31647.2667 \\
\hline $\mathbf{1 5}$ & $\mathbf{1 2 8 0 . 6 0}$ & $\mathbf{1 4 1 9 . 3 2 3}$ & $\mathbf{4 7 7 1 . 7 9 8}$ & $\mathbf{8 6 9 . 6 8 3 1}$ \\
\hline 16 & 65158.35 & 54334.791 & 64416.288 & 68496.7309 \\
\hline
\end{tabular}

paths obtained with the other smartphones. In fact, the second closest path provides a DTW around 25 times higher that the shortests distance, which means that the DTW distance really fit for zone detection with magnetic field.

In general, the obtained results show that magnetic field can be linked to every one of the "virtual corridors" defined.

\section{Conclusions}

In this paper we have shown the situation of Parc Taulí Hospital (PTH) for getting positioning inside the common and open areas of the hospital. It is a challenging situation because: 1) it combines wide spaces like the hall or the reception with corridors and middle size spaces like waiting rooms; 2) it is a shielded zone where no signals are available, therefore, no WiFi signal can be used for positioning; and 3) it is not possible to deploy neither beacons nor sensors for positioning. On the other hand, the hospital only needs to know the zone where a single user is, and not his or her exact location with centimeter accuracy.

In the presented work, we tested whether the magnetic field could be an appropriate signal to find position in the PTH environment. However, magnetic field has proven as an effective tool for positioning users when moving, but not for static positions; and when users are moving along corridors. Since the anomalies of the magnetic field that lead to a position are originated by structures of the building or fixed devices, they are usually too weak to position users in wide free areas. Thus, obtaining the paths in corridors may be straightforward, but in wide areas is still challenging. With the purpose of addressing such challenge, we have created "virtual corridors" that simulate the expected displacements of users when get into this particular hospital.

We have collected magnetic data following those "virtual corridors" as well as the real corridors in a typical user experience. Data have been obtained from five different smartphones, with different hardware and OS version, and the paths have been followed in both directions. To verify if the second requirement is satisfied, data obtained from all the paths from every single smartphone, have been compared with all the paths obtained from the other smartphones. Comparison have been performed using dynamic time warping algorithm.

The results obtained have shown that $100 \%$ of cases compared, the closer path to a single one, is the corresponding path obtained with the other smartphones. That shows that despite the wide spaces that can be found in the hospital, magnetic field is a promising mechanism of positioning when only the zone where users is need to be located.

The main contributions of the present paper are: 1) defining "virtual corridors" as a way to position using magnetic field in 2D spaces; 2) showing that even in wide spaces like the hall of a hospital, it is possible to find magnetic anomalies linked to positions.

Finally, this paper shows an application of indoor positioning of low requirements regarding precision, showing that there are useful applications for indoor positioning where precision is not the main requirement. Therefore, it is possible to start deploying simple applications that look for some kind of standardization, even in low precision scenarios. This can help to expand indoor applications and indoor positioning functionalities. This is similar to the process followed by GNSS that, at the beginning offered low precision to end users, but its popularization has helped to find standards and increase precision.

As a future work we plan to look for the minimum path needed to find the position of the user and which is the minimum area in which the user can be localized, i.e. what is the position of the user inside the path. That will allow us to know the amount of time needed to get data. On the other hand, while DTW accounts for different velocity in traversing the path, we plan to analyse what happens when the actual path is slightly different from the stored path and what is the amount of deviation that is tolerable.

\section{ACKNOWLEDGEMENTS}

Authors want to thank the collaboration of REPNIN spanish excellence network in indoor positioning and navigation. TEC2015-71426-REDT. And also to Parc Taulí Hospital Foundation for its collaboration in the development of this experiment. One of us, Dr. Raul Montoliu wants to thank Spanish Ministry of Economy and Competitiveness in the project: Proyectos I+D Excelencia TIN2015-70202-P.

\section{REFERENCES}

[1] D. Lymberopoulos, J. Liu, X. Yang, R. R. Choudhury, V. Handziski, and S. Sen, "A realistic evaluation and comparison of indoor location technologies," in Proceedings of the 14th International Conference on Information Processing in Sensor Networks - IPSN '15. New York, New York, USA: ACM Press, 2015, pp. 178-189. [Online]. Available: http://dl.acm.org/citation.cfm?doid=2737095.2737726

[2] J. Torres-Sospedra, A. Jiménez, A. Moreira, T. Lungenstrass, W.-C. Lu, S. Knauth, G. Mendoza-Silva, F. Seco, A. Pérez-Navarro, M. Nicolau, A. Costa, F. Meneses, J. Farina, J. Morales, W.-C. Lu, H.-T. Cheng, S.-S. Yang, S.-H. Fang, Y.-R. Chien, and Y. Tsao, "Off-Line Evaluation of Mobile-Centric Indoor Positioning Systems: The Experiences from the 2017 IPIN Competition," Sensors, vol. 18, no. 2, p. 487, feb 2018. [Online]. Available: http://www.mdpi.com/1424-8220/18/2/487

[3] N. Newman, "Apple iBeacon technology briefing," Journal of Direct, Data and Digital Marketing Practice, vol. 15, 2014. 
[4] C. Koehler, N. Banovic, I. Oakley, J. Mankoff, and A. K. Dey, "IndoorALPS," in Proceedings of the 2014 ACM International Joint Conference on Pervasive and Ubiquitous Computing - UbiComp '14 Adjunct. New York, New York, USA: ACM Press, 2014, pp. 171-181. [Online]. Available: http://dl.acm.org/citation.cfm?doid=2632048.2632069

[5] L. Li, P. Hu, C. Peng, G. Shen, and F. Zhao, "Epsilon: A visible light based positioning system," 11th USENIX Symposium on Networked Systems Design and Implementation (NSDI 14), pp. 331-343, 2014.

[6] T. Ye, M. Walsh, P. Haigh, J. Barton, A. Mathewson, and B. O'Flynn, "An Experimental Evaluation of IEEE 802.15.4a Ultra Wide Band Technology for Precision Indoor Ranging," International Journal of Ambient Computing and Intelligence, vol. 4, no. 2, pp. 48-63, 2012. [Online]. Available: http://services.igi-global.com/resolvedoi/resolve. aspx?doi=10.4018/jaci.2012040104

[7] E. Laitinen and E. Lohan, "On the Choice of Access Point Selection Criterion and Other Position Estimation Characteristics for WLAN-Based Indoor Positioning," Sensors, vol. 16, no. 5, p. 737, may 2016. [Online] Available: http://www.mdpi.com/1424-8220/16/5/737

[8] World Health Organization, "Electromagnetic fields and public health mobile phones," Available at http://www.who.int/news-room/fact-sheets/ detail/electromagnetic-fields-and-public-health-mobile-phones, 2014.

[9] A. Solin, M. Kok, N. Wahlstrom, T. B. Schon, and S. Sarkka, "Modeling and Interpolation of the Ambient Magnetic Field by Gaussian Processes,' IEEE Transactions on Robotics, pp. 1-16, 2018. [Online]. Available https://ieeexplore.ieee.org/document/8373720/

[10] T. Bagosi and Z. Baruch, "Indoor localization by wifi," in IEEE International Conference on Intelligent Computer Communication and Processing (ICCP), 2011, pp. 449-452.

[11] R. Montoliu, J. Torres-Sospedra, and O. Belmonte, "Magnetic field based Indoor positioning using the Bag of Words paradigm," in 2016 International Conference on Indoor Positioning and Indoor Navigation (IPIN). IEEE, oct 2016, pp. 1-7. [Online]. Available: http://ieeexplore.ieee.org/document/7743596/

[12] D. Carrillo, V. Moreno, B. Úbeda, and A. Skarmeta, "MagicFinger: 3D Magnetic Fingerprints for Indoor Location," Sensors, vol. 15, no. 7, pp. $17168-17194$, jul 2015. [Online]. Available: http: //www.mdpi.com/1424-8220/15/7/17168/

[13] S. Shahidi and S. Valaee, "GIPSy: Geomagnetic indoor positioning system for smartphones," in 2015 International Conference on Indoor Positioning and Indoor Navigation (IPIN). IEEE, oct 2015, pp. 1-7. [Online]. Available: http://ieeexplore.ieee.org/document/7346761/

[14] C. Galván-Tejada, J. García-Vázquez, and R. Brena, "Magnetic Field Feature Extraction and Selection for Indoor Location Estimation," Sensors, vol. 14, no. 6, pp. 11001-11015, jun 2014. [Online]. Available: http://www.mdpi.com/1424-8220/14/6/11001/

[15] A. Bilke and J. Sieck, "Using the Magnetic Field for Indoor Localisation on a Mobile Phone," 2013, pp. 195-208. [Online]. Available: http://link.springer.com/10.1007/978-3-642-34203-5\{\_\}11

[16] H.-S. Kim, W. Seo, and K.-R. Baek, "Indoor Positioning System Using Magnetic Field Map Navigation and an Encoder System," Sensors, vol. 17, no. 3, p. 651, mar 2017. [Online]. Available: http://www.mdpi.com/1424-8220/17/3/651

[17] J. Haverinen and A. Kemppainen, "A global self-localization technique utilizing local anomalies of the ambient magnetic field," in 2009 IEEE International Conference on Robotics and Automation. IEEE, may 2009, pp. 3142-3147. [Online]. Available: http://ieeexplore.ieee.org/document/5152885/

[18] P. Jiang, Y. Zhang, W. Fu, H. Liu, and X. Su, "Indoor Mobile Localization Based on Wi-Fi Fingerprint's Important Access
Point," International Journal of Distributed Sensor Networks, vol. 11, no. 4, p. 429104, apr 2015. [Online]. Available: http: //journals.sagepub.com/doi/10.1155/2015/429104

[19] Y. Kim, Y. Chon, and H. Cha, "Smartphone-Based Collaborative and Autonomous Radio Fingerprinting," IEEE Transactions on Systems, Man, and Cybernetics, Part C (Applications and Reviews), vol. 42, no. 1, pp. 112-122, jan 2012. [Online]. Available: http://ieeexplore.ieee.org/document/5675696/

[20] Yiqiang Chen, Qiang Yang, Jie Yin, and Xiaoyong Chai, "Power-efficient access-point selection for indoor location estimation," IEEE Transactions on Knowledge and Data Engineering, vol. 18, no. 7, pp. 877-888, jul 2006. [Online]. Available: http://ieeexplore.ieee.org/document/1637415/

[21] K. Li, J. Bigham, E. L. Bodanese, and L. Tokarchuk, "Location estimation in large indoor multi-floor buildings using hybrid networks,' in 2013 IEEE Wireless Communications and Networking Conference (WCNC). IEEE, apr 2013, pp. 2137-2142. [Online]. Available: http://ieeexplore.ieee.org/document/6554893/

[22] B. Gozick, K. Pathapati Subbu, R. Dantu, and T. M. Maeshiro, "Magnetic Maps for Indoor Navigation," IEEE TRANSACTIONS ON INSTRUMENTATION AND MEASUREMENT, vol. 60, no. 12 , p. 8 , 2011.

[23] K. P. Subbu, B. Gozick, and R. Dantu, "Locateme: Magnetic-fields-based indoor localization using smartphones," ACM Trans. Intell. Syst. Technol., vol. 4, no. 4, pp. 73:1-73:27, Oct. 2013. [Online]. Available: http://doi.acm.org/10.1145/2508037.2508054

[24] M. Bennewitz, W. Burgard, G. Cielniak, and S. Thrun, "Learning Motion Patterns of People for Compliant Robot Motion," The International Journal of Robotics Research, vol. 24, no. 1, pp. 31-48, jan 2005. [Online]. Available: http://journals.sagepub.com/doi/10.1177/0278364904048962

[25] B. Kuipers, "The Spatial Semantic Hierarchy," Artificial Intelligence, vol. 119, no. 1-2, pp. 191-233, may 2000. [Online]. Available: http://linkinghub.elsevier.com/retrieve/pii/S0004370200000175

[26] T. Ikeda, Y. Chigodo, D. Rea, F. Zanlungo, M. Shiomi, and T. Kanda, "Modeling and prediction of pedestrian behavior based on the sub-goal concept." in Robotics: Science and Systems, 2012. [Online]. Available: http://dblp.uni-trier.de/db/conf/rss/rss2012.html\#IkedaCRZSK12

[27] "The GetSensorData Android App for registering all sensor stream in a smartphone," https://lopsi.weebly.com/downloads.html.

[28] A. R. Jiménez, F. Zampella, and F. Seco, "Light-Matching : a new Signal of Opportunity for Pedestrian Indoor Navigation," in International Conference on Indoor Positioning and Indoor Navigation (IPIN), vol. 1, no. October, Montbeliard-Belfort, 2013, pp. 777-786.

[29] F. Potortì, S. Park, A. R. Jiménez Ruiz, P. Barsocchi, M. Girolami, A. Crivello, S. Y. Lee, J. H. Lim, J. Torres-Sospedra, F. Seco, R. Montoliu, G. M. Mendoza-Silva, M. D. C. Pérez Rubio, C. Losada-Gutiérrez, F. Espinosa, and J. Macias-Guarasa, "Comparing the performance of indoor localization systems through the evaal framework," Sensors, vol. 17 , no. 10,2017

[30] P. Tormene, T. Giorgino, S. Quaglini, and M. Stefanelli, "Matching incomplete time series with dynamic time warping: an algorithm and an application to post-stroke rehabilitation," Artificial Intelligence in Medicine, vol. 45, no. 1, pp. 11-34, jan 2009. [Online]. Available: http://linkinghub.elsevier.com/retrieve/pii/S0933365708001772

[31] T. Giorgino, "Computing and Visualizing Dynamic Time Warping Alignments in R : The dtw Package," Journal of Statistical Software, vol. 31, no. 7, 2009. [Online]. Available: http://www.jstatsoft.org/v31/i07/ 